\title{
Relationship between clinical types and radiological subgroups defined by latent class analysis in 2019 novel coronavirus pneumonia caused by SARS-CoV-2
}

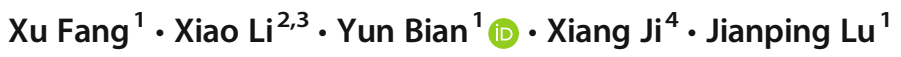 \\ Received: 28 March 2020 / Revised: 5 May 2020 / Accepted: 20 May 2020 / Published online: 30 May 2020 \\ (C) European Society of Radiology 2020
}

\begin{abstract}
Objectives To investigate whether meaningful subgroups sharing the CT features of patients with COVID-19 pneumonia could be identified using latent class analysis (LCA) and explore the relationship between the LCA-derived subgroups and clinical types.

Methods This retrospective review included 499 patients with confirmed COVID-19 pneumonia between February 11 and March 8, 2020. Subgroups sharing the CT features were identified using LCA. Univariate and multivariate logistic regression models were utilized to analyze the association between clinical types and the LCA-derived subgroups.

Results Two radiological subgroups were identified using LCA. There were 228 subjects (45.69\%) in class 1 and 271 subjects $(54.31 \%)$ in class 2 . The CT findings of class 1 were smaller pulmonary infection volume, more peripheral distribution, more GGO, more maximum lesion range $\leq 5 \mathrm{~cm}$, a smaller number of lesions, less involvement of lobes, less air bronchogram, less dilatation of vessels, less hilar and mediastinal lymph node enlargement, and less pleural effusion than the CT findings of class 2. Univariate analysis demonstrated that older age, therapy, presence of fever, presence of hypertension, decreased lymphocyte count, and increased CRP levels were significant parameters associated with an increased risk for class 2 . Multivariate analyses revealed that the patients with clinically severe type disease had a 1.97-fold risk of class 2 than the patients with clinically moderate-type disease.

Conclusions The demographic and clinical differences between the two radiological subgroups based on the LCA were significantly different. Two radiological subgroups were significantly associated with clinical moderate and severe types.

Key Points

- Two radiological subgroups were identified using LCA.

- Older age, therapy, presence offever, presence of hypertension, decreased lymphocyte count, and increased CRP levels were significant parameters with an increased risk for class 2 defined by LCA.

- Patients with clinically severe type had a 1.97-fold higher risk of class 2 defined by LCA in comparison with patients showing clinically moderate-type disease.
\end{abstract}

Keywords Coronavirus infections · Tomography, X-ray computed · Pneumonia, Latent class analysis

Xu Fang and Xiao Li are the joint first authors in the paper.

Yun Bian

bianyun2012@foxmail.com

1 Department of Radiology, Changhai Hospital, The Navy Military Medical University, Changhai road 168, Shanghai 200434, China

2 Department of Medical Imaging, Jinling Hospital, Medical School of Nanjing University, Nanjing 210002, Jiangsu, China

3 Department of Radiology, Wuhan Huoshenshan Hospital, Wuhan 430000, Hubei, China

4 Shanghai United Imaging Intelligence Healthcare, Shanghai, China

$\begin{array}{ll}\text { Abbreviations } & \\ \text { 2019-nCoV } & \text { 2019-novel coronavirus } \\ \text { BMI } & \text { Body mass index } \\ \text { CI } & \text { Confidence interval } \\ \text { CRP } & \text { C-reactive protein } \\ \text { GGO } & \text { Ground glass opacity } \\ \text { HRCT } & \text { High-resolution CT } \\ \text { LCA } & \text { Latent class analysis } \\ \text { MERS } & \text { Middle East respiratory syndrome } \\ \text { SARS } & \text { Severe acute respiratory syndrome } \\ \text { WHO } & \text { World Health Organization }\end{array}$




\section{Introduction}

In December 2019, a succession of cases of pneumonia with unknown causes appeared in Wuhan, Hubei Province, China. On January 7, 2020, the 2019 novel coronavirus (with the virus officially named by the World Health Organization as SARS-CoV-2) was identified as the causative agent based on virus typing $[1,2]$. Recent studies have revealed that the SARS-CoV-2 is more closely related to bat-SL-CoV ZC45 and bat-SL-CoV ZXC21 [2] and shows human-to-human spread mainly through respiratory droplets, aerosol, contact, and the oral-fecal route [3]. At present, the infection has spread across China and other countries around the world [4-6].

The National Health Commission of China [7, 8] formulated the Diagnosis and Treatment Program of 2019 New Coronavirus Pneumonia (trial seventh version) based on the recommendations of the World Health Organization (WHO) on severe acute respiratory syndrome (SARS) and Middle East respiratory syndrome (MERS) [9-11]. In the trial seventh version, the clinical type was classified into mild, moderate, severe, and critical types according to the clinical manifestations (Table 1). The mild type was defined as mild clinical symptoms with negative imaging findings, and the critical type was defined as respiratory failure, shock, and "extrapulmonary" organ failure. The patients with critical COVID-19 pneumonia were hard to undergo CT scan in intensive care unit. Thus, positive CT findings were often found in patients with moderate and severe disease. Patients with mild and moderate disease have a good prognosis. However, when COVID-19 pneumonia develops to severe and critical levels, pulmonary edema, respiratory failure, shock, and multiple organ failure can eventually cause death. Therefore, it is very important to accurately and easily classify the CT findings and administer rapid clinical interventions accordingly.
Numerous CT findings almost always co-exist in all clinical types. Thus, multiple CT findings may be more strongly associated with clinical types than single CT findings. Latent class analysis (LCA) has recently been tested and found to be useful for identifying latent classes (subgroups) of CT findings within multivariable datasets. However, a classification of individual patients with COVID-19 pneumonia based purely on CT characteristics has not been presented so far. Hence, the primary objective of our study was to investigate whether meaningful subgroups sharing the $\mathrm{CT}$ features of patients with COVID-19 pneumonia could be identified using latent class analysis (LCA) and explore the relationship between the LCA-derived subgroups and clinical types (moderate and severe types).

\section{Methods}

\section{Patients}

This retrospective cross-sectional study was reviewed and approved by the Biomedical Research Ethics Committee of our institution, and the requirement for patient consent was waived.

The inclusion criteria were (1) the availability of a positive reverse-transcription polymerase chain reaction (RT-PCR) tests confirming the viral origin of the pneumonia and (2) the availability of a chest $\mathrm{CT}$ at the time of diagnosis. The exclusion criteria were (1) patients who had not been confirmed by RT-PCR tests, (2) patients who had not undergone pulmonary HRCT, (3) normal lung parenchyma on chest CT, (4) presence of non-infectious lung parenchyma lesions on chest CT (e.g., lung cancer, pneumothorax, pulmonary ede$\mathrm{ma}$ ), and (5) a delay between chest $\mathrm{CT}$ and confirmation of the clinical type longer than 3 days. Finally, we retrospectively identified 499 consecutive patients with COVID-19

Table 1 The clinical classification of COVID-19 pneumonia from the 7th edition of the National Commission of China classification

\begin{tabular}{ll}
\hline Types & Findings \\
\hline Mild & $\begin{array}{l}\text { Mild clinical symptoms (fever }<38{ }^{\circ} \mathrm{C} \text { (quelled without treatment), with or without cough, no dyspnea, no gasping, no chronic disease) } \\
\text { *No imaging findings of pneumonia }\end{array}$ \\
Moderate & Fever, respiratory symptoms \\
& *Imaging findings of pneumonia \\
Severe & $\begin{array}{l}\text { Meet any of the followings: } \\
\text { (a) Respiratory distress, RR } \geq 30 \text { times } / \text { min }\end{array}$ \\
& (b) SpO2 $<93 \%$ at rest \\
(c) PaO2/FiO2 $\leq 300 \mathrm{mmHg}$ \\
*Patients showing a rapid progression ( $>50 \%$ ) on CT imaging within $24-48$ h should be managed as severe (added in the trial sixth edition) \\
Meet any of the followings: \\
(a) Respiratory failure, need mechanical assistance \\
(b) Shock \\
(c) "Extra pulmonary" organ failure, intensive care unit is needed
\end{tabular}

*CT findings 
pneumonia at Wuhan Huoshenshan Hospital between February 11 and March 8, 2020. All clinical results were extracted from the patients' electronic medical records in the hospital information system (Fig. 1).

\section{CT scanning}

Pulmonary CT was performed using 128-slice multidetector row CT scanners (uCT 760, United Imaging Healthcare, respectively). CT scans were obtained with the following parameters: $120 \mathrm{kV}$, adaptive tube current, beam collimation of $128 \times 0.6 \mathrm{~mm}$, and a $512 \times 512$ matrix. A non-enhanced CT was performed. The slice thickness was $0.625 \mathrm{~mm}$, respectively. Images were captured at window settings that allowed viewing of the lung parenchyma (window level, -600 to $700 \mathrm{HU}$; window width, 1200-1500 HU) and the mediastinum (window level, 20-40 HU; window width, $400 \mathrm{HU}$ ). The scanning range was from the level of the superior aperture of thorax to the diaphragm.

\section{Radiological imaging analysis}

We used the original cross-sectional images for analysis. All the images were analyzed by two abdominal radiologists (X.L. and X.F., with 8 years of experience each) who were blinded to the clinical details; the final results were determined by consensus.

All lesions were evaluated for the following characteristics: (a) pulmonary infection volume: total, left, and right pulmonary infection volume, which was calculated by artificial intelligence software (uAI-Discover-NCP R001, United Imaging Healthcare); (b) location: right, left, or bilateral; (c) distribution: peripheral, central, or diffuse distribution; (d) attenuation: ground glass attenuation including ground glass opacity (GGO), crazy-paving pattern, consolidation, or mixed pattern $[12,13]$; (e) maximum lesion range: $\leq 5 \mathrm{~cm}, 5-10 \mathrm{~cm}$, or $>10 \mathrm{~cm}$ only for the biggest one; (f) involvement of lobes; (g) number of lesions: 1, 2, 3, or more; (h) air bronchogram; (i) dilatation of vessels; (j) hilar lymphadenopathy: short-axis

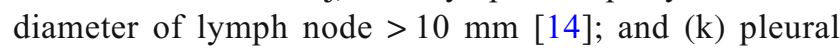
effusion.

\section{Statistical analyses}

Normal distribution and variance homogeneity tests were performed on all continuous variables; those with a normal distribution were expressed as the mean and standard deviation while those with non-normal distributions were expressed as medians and ranges. First, LCA was performed using R software supplemented with the mclust package. LCA is a statistical method in which a set of multivariate data are used to identify groups of related subjects ("latent classes") within data that share similar characteristics. The Akaike information criteria (AIC) were used to identify the optimum number of classes in the model as the number yielding the lowest AIC value [15]. Second, all patients were divided into LCA-derived subgroups. We examined subgroup differences in all variables. Kruskal-Wallis H test (skewed distribution) and the chi-squared test (categorical variables) were used to determine the statistical differences among the subgroups. Third, univariate regression analysis was applied to estimate effect sizes for the relationships between all variables and LCA-derived subgroups. Finally, multivariable logistic models were used to evaluate the
Fig. 1 Flowchart visualizing the patient selection process

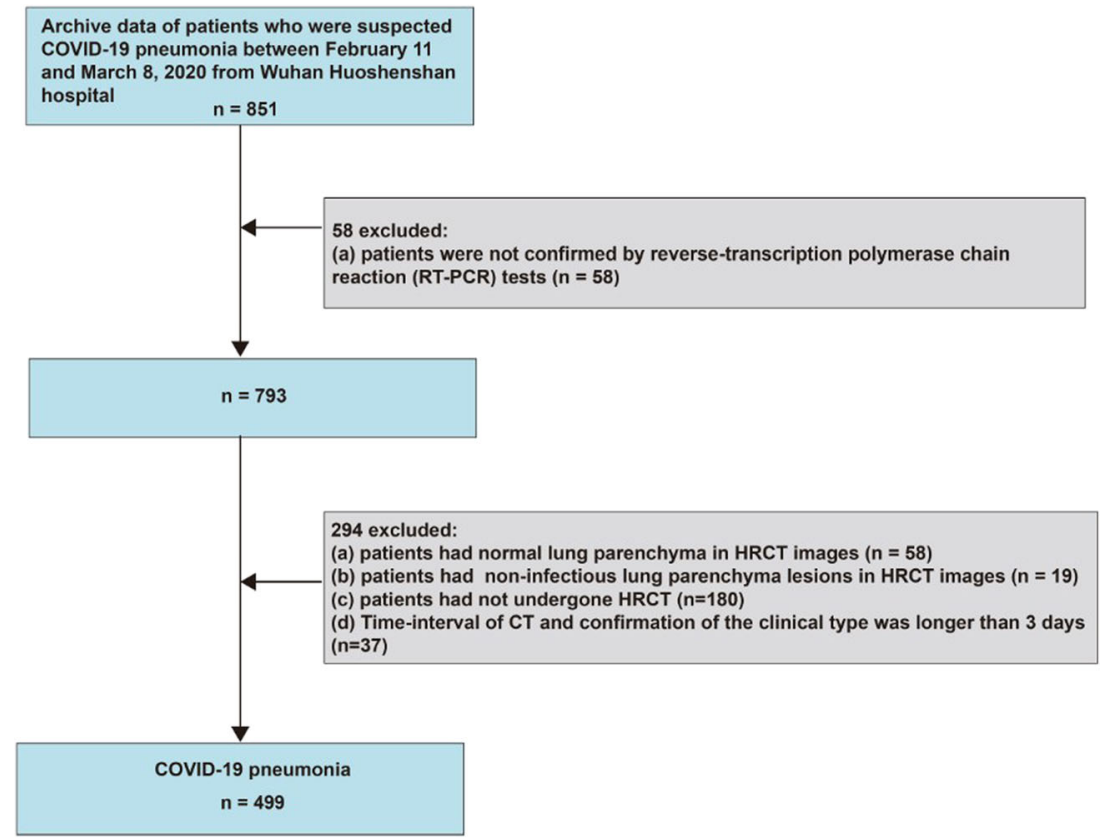




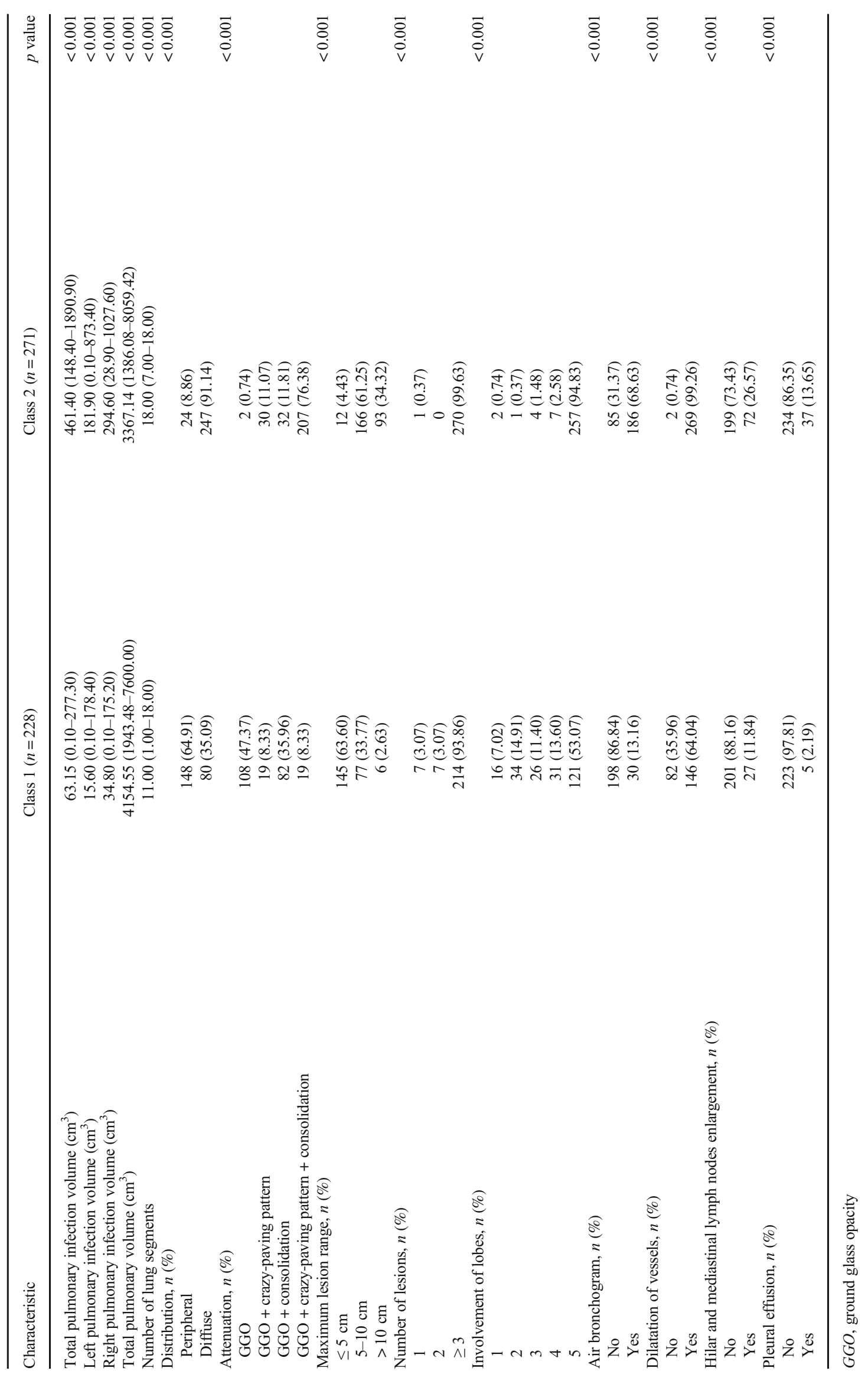


Fig. 2 CT findings (continuous variables) of latent classes identified

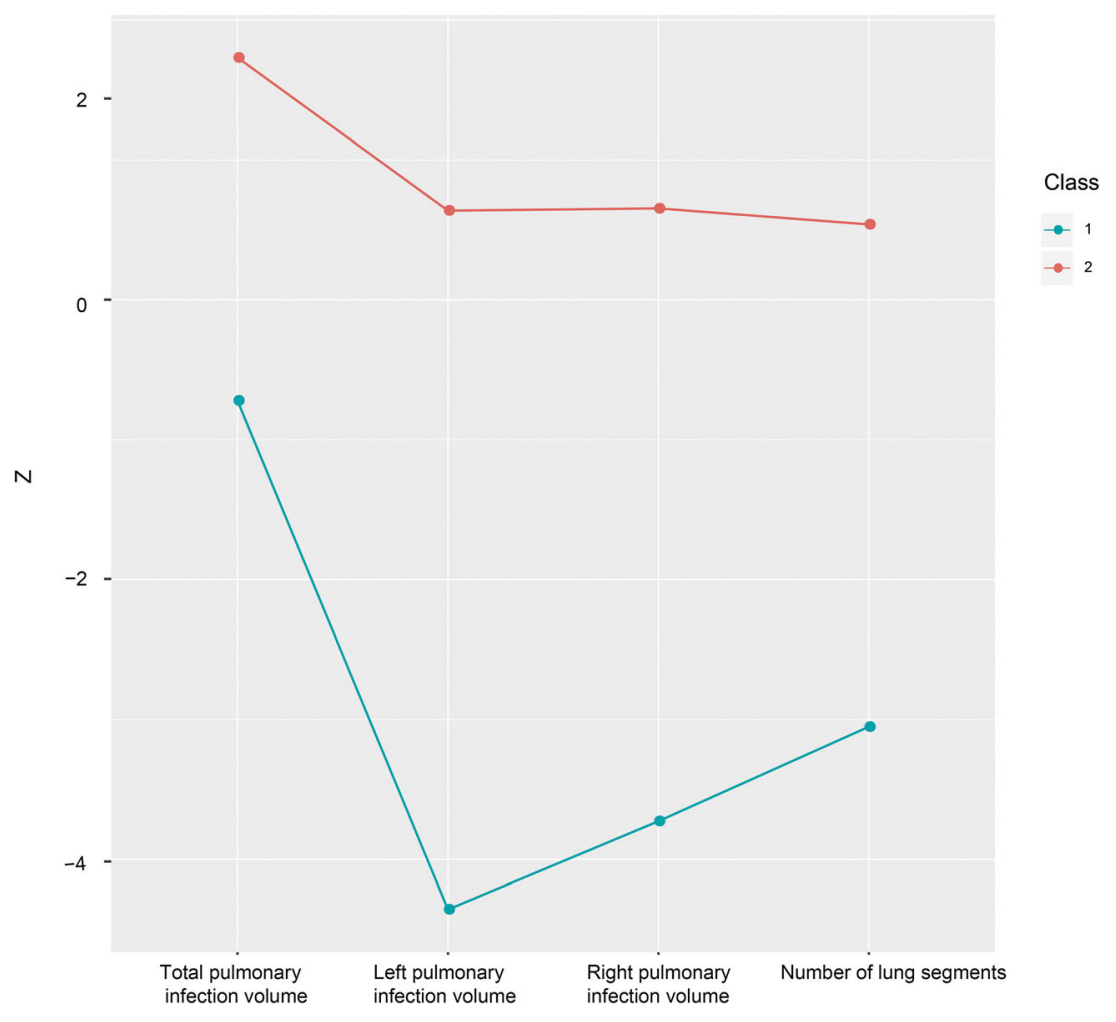

associations between exposure (clinical characteristics) and outcome (LCA-derived subgroups). These models included model 1 (not adjusted for other covariates), model 2 (adjusted for age, sex, and body mass index [BMI]), and model 3 (adjusted for the same factors as model 2 as well as for other significantly associated clinical and imaging characteristics in univariate regression analysis). Moderate of the clinical types was considered as the reference group.

A two-tailed $p$ value less than 0.05 was considered statistically significant. All analyses were performed using R software (version 3.6.1, The R Foundation for Statistical Computing).

\section{Results}

\section{Identification of latent classes}

Two latent classes were identified using the AIC. There were 228 subjects $(45.69 \%)$ in class 1 and $271(54.31 \%)$ in class 2. The CT features of each of the latent classes are shown in Table 2 and Figs. 2 and 3. The CT findings of class 1 were smaller pulmonary infection volume, more peripheral distribution, more GGO, more maximum lesion range $\leq 5 \mathrm{~cm}$, a smaller number of lesions, less involvement of lobes, less air bronchogram, less dilatation of vessels, less hilar and mediastinal lymph node enlargement, and less pleural effusion than the CT findings of class 2 .

\section{Demographic and clinical differences}

The demographic and clinical characteristics of the LCAderived subgroups are shown in Table 2. Among the characteristics that we investigated, age, outcomes, fever, hypertension, lymphocyte count, and C-reactive protein (CRP) level were significantly different (Table 3).

\section{Univariate analysis of LCA-derived subgroups and clinical characteristics}

The univariate analysis results are shown in Table 4, demonstrating that older age $(p<0.0001)$, therapy of outcomes $(p<0.0001)$, presence of fever $(p=0.001)$, presence of hypertension ( $p=0.006)$, decreased lymphocyte count $(p=0.014)$, and increased CRP levels $(p<0.0001)$ were significant parameters with an increased risk for class 2 defined by LCA (Fig. 4).

\section{Multivariate analyses of the LCA-derived subgroups and clinical types}

Multivariable logistic models were used to evaluate the associations between exposure (clinical types) and outcome (the LCA-derived subgroups). In the crude model (model 1), clinical types were correlated with the LCA-derived subgroups (odds ratio [OR] 3.49, 95\% confidence interval [CI] 2.265.39 ], $p<0.0001$ ). In the minimally adjusted model (adjusted 

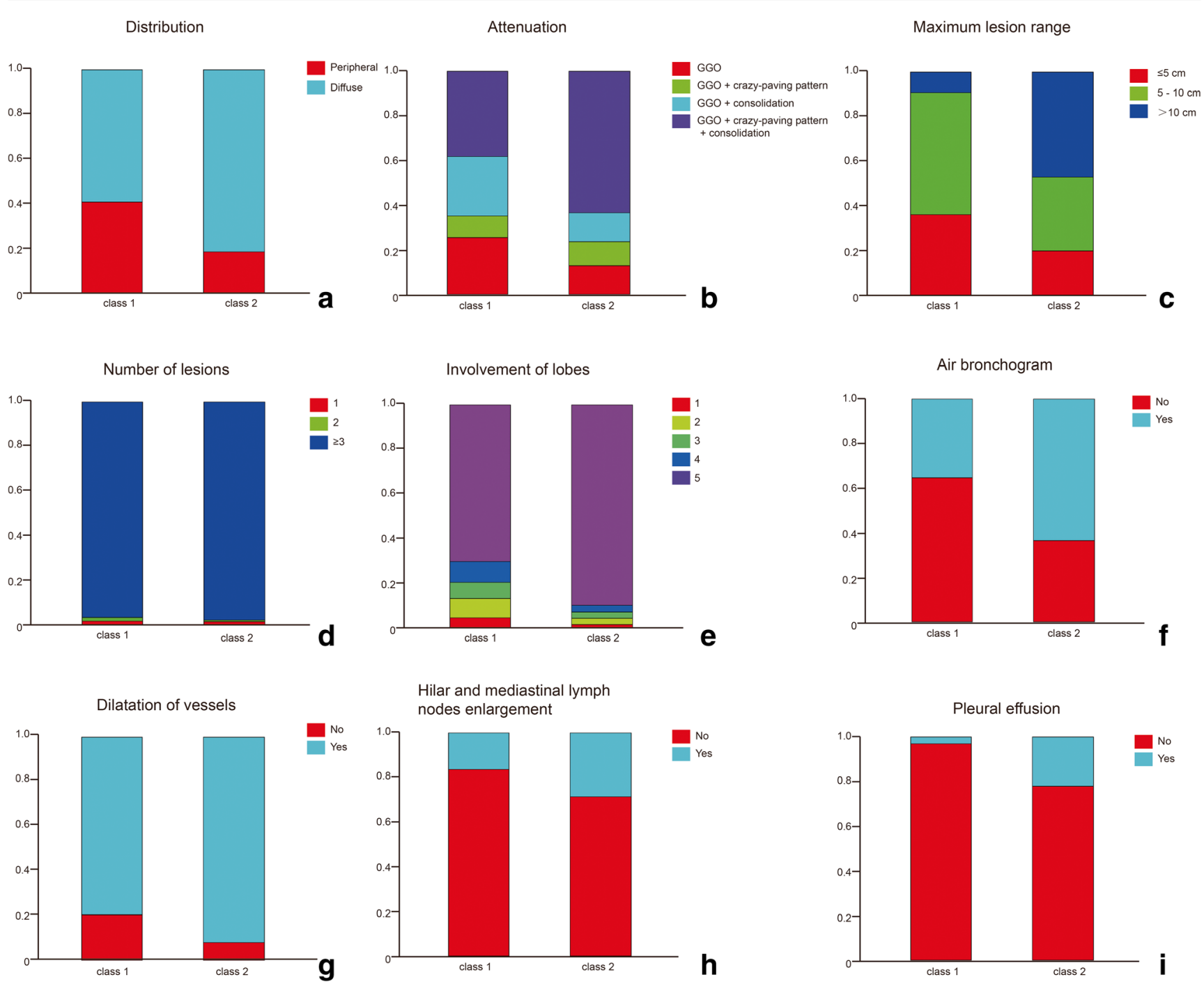

Fig. 3 CT findings (categorical variables) of lung segments of latent classes identified

for age, sex, and BMI) (model 2), the effect size also showed a significant correlation (OR 3.01, 95\% CI 1.92-4.70, $p<0.0001$ ). After further adjustment for outcome, fever, hypertension, lymphocyte count, and CRP levels, significance was still identified in the fully adjusted model (model 3) (OR $1.97,95 \%$ CI $1.09-3.54, p=0.025$ ). The results of multivariate analysis are shown in Table 5.

\section{Discussion}

The present study aimed to investigate meaningful subgroups sharing the CT features of patients with COVID-19 pneumonia that could be identified using LCA, and to explore the relationship between the LCA-derived subgroups and clinical types. Two latent classes were identified using LCA. Furthermore, in the fully adjusted model (model 3), the LCA-derived subgroups were significantly associated with clinical types.
In the current study, we found that class 1 (median 4154.55 , range $1943.48-7600.00 \mathrm{~cm}^{3}$ ) was significantly larger than class 2 (median 3367.14, range $1386.08-8059.42 \mathrm{~cm}^{3}$ ) in the total pulmonary volume. The current results are consistent with the findings of the previous studies. Iwasawa et al. [16] found smaller CT lung volume in severe cases was observed, and ultra-high-resolution CT showed that secondary lobes in the crazy-paving pattern were smaller than in unaffected lungs. Wu et al. [17] reported that these lesions frequently pulled the adjacent pleura. Allbarello et al. [18] found decreased normal lung volume in COVID-19 pneumonia patients with acute respiratory distress syndrome. These results indicated that pulmonary fibrosis destroyed the alveoli leading to the local volume loss, which was more common in severe than moderate of COVID-19 pneumonia.

A few studies have explored the relationship between CT and clinical characteristics and disease severity. Wu et al. [17] used the pulmonary inflammation index (PII) value to evaluate the relationship between CT findings and clinical features, 
Table 3 Clinical characteristics of latent classes analysis

\begin{tabular}{|c|c|c|c|}
\hline Characteristic & Class $1(n=228)$ & Class $2(n=271)$ & $p$ value \\
\hline Sex, $n(\%)$ & & & 0.485 \\
\hline Male & $114(50.00)$ & $144(53.14)$ & \\
\hline Female & $114(50.00)$ & $127(46.86)$ & \\
\hline Age, mean (SD), years & $53.90 \pm 15.07$ & $61.12 \pm 12.52$ & $<0.001$ \\
\hline BMI, mean (SD), $\mathrm{kg} / \mathrm{m}^{2}$ & $22.76 \pm 3.00$ & $22.61 \pm 3.29$ & 0.595 \\
\hline Clinical type, $n(\%)$ & & & $<0.001$ \\
\hline Moderate & $193(84.65)$ & $166(61.25)$ & \\
\hline Severe & $35(15.35)$ & $105(38.75)$ & \\
\hline Initial nucleic acid test, $n(\%)$ & & & 0.789 \\
\hline Negative & $13(5.70)$ & $17(6.27)$ & \\
\hline Positive & $215(94.30)$ & $254(93.73)$ & \\
\hline Outcomes, $n(\%)$ & & & $<0.001$ \\
\hline Recovery & $212(92.98)$ & $210(77.49)$ & \\
\hline Therapy & $16(7.02)$ & $53(19.56)$ & \\
\hline Death & 0 & $8(2.95)$ & \\
\hline Fever, $n(\%)$ & & & $<0.001$ \\
\hline No & $46(20.18)$ & $26(9.59)$ & \\
\hline Yes & $182(79.82)$ & $245(90.41)$ & \\
\hline Cough, $n(\%)$ & & & 0.249 \\
\hline No & $57(25.00)$ & $56(20.66)$ & \\
\hline Yes & $171(75.00)$ & $215(79.34)$ & \\
\hline Myalgia or arthralgia, $n(\%)$ & & & 0.172 \\
\hline No & $140(61.40)$ & $150(55.35)$ & \\
\hline Yes & $88(38.60)$ & $121(44.65)$ & \\
\hline Headache, $n(\%)$ & & & 0.973 \\
\hline No & $222(97.37)$ & $264(97.42)$ & \\
\hline Yes & $6(2.63)$ & $7(2.58)$ & \\
\hline Nausea or vomiting, $n(\%)$ & & & 0.831 \\
\hline No & $225(98.68)$ & $268(98.89)$ & \\
\hline Yes & $3(1.32)$ & $3(1.11)$ & \\
\hline Diarrhea, $n(\%)$ & & & 0.849 \\
\hline No & $213(93.42)$ & $252(92.99)$ & \\
\hline Yes & $15(6.58)$ & $19(7.01)$ & \\
\hline Abdominal pain, $n(\%)$ & & & 0.039 \\
\hline No & $228(100.00)$ & $266(98.15)$ & \\
\hline Yes & $0(0.00)$ & $5(1.85)$ & \\
\hline Smoking history, $n(\%)$ & & & 0.140 \\
\hline No & $217(95.18)$ & $249(91.88)$ & \\
\hline Yes & $11(4.82)$ & $22(8.12)$ & \\
\hline Cardiovascular disease, $n(\%)$ & & & 0.884 \\
\hline No & $207(90.79)$ & $245(90.41)$ & \\
\hline Yes & $21(9.21)$ & $26(9.59)$ & \\
\hline Diabetes, $n(\%)$ & & & 0.079 \\
\hline No & 209 (91.67) & $235(86.72)$ & \\
\hline Yes & $19(8.33)$ & $36(13.28)$ & \\
\hline Hypertension, $n(\%)$ & & & 0.006 \\
\hline No & $173(75.88)$ & $175(64.58)$ & \\
\hline Yes & $55(24.12)$ & $96(35.42)$ & \\
\hline
\end{tabular}


Table 3 (continued)

\begin{tabular}{|c|c|c|c|}
\hline Characteristic & Class $1(n=228)$ & Class $2(n=271)$ & $p$ value \\
\hline COPD, $n(\%)$ & & & 0.902 \\
\hline No & $227(99.56)$ & $270(99.63)$ & \\
\hline Yes & $1(0.44)$ & $1(0.37)$ & \\
\hline Chronic liver disease, $n(\%)$ & & & 0.987 \\
\hline No & $223(97.81)$ & $265(97.79)$ & \\
\hline Yes & $5(2.19)$ & $6(2.21)$ & \\
\hline Chronic renal disease, $n(\%)$ & & & 0.540 \\
\hline No & $224(98.25)$ & $268(98.89)$ & \\
\hline Yes & $4(1.75)$ & $3(1.11)$ & \\
\hline Malignant tumor, $n(\%)$ & & & 0.596 \\
\hline No & $223(97.81)$ & $263(97.05)$ & \\
\hline Yes & $5(2.19)$ & $8(2.95)$ & \\
\hline WBC count, $n(\%)$ & & & 0.067 \\
\hline Decreased & $27(11.84)$ & $19(7.01)$ & \\
\hline Normal & $191(83.77)$ & $231(85.24)$ & \\
\hline Increased & $10(4.39)$ & $21(7.75)$ & \\
\hline Lymphocyte count, $n(\%)$ & & & $<0.001$ \\
\hline Increased & $6(2.63)$ & $2(0.74)$ & \\
\hline Normal & $165(72.37)$ & $123(45.39)$ & \\
\hline Decreased & $57(25.00)$ & $146(53.87)$ & \\
\hline $\mathrm{CRP}, n(\%)$ & & & $<0.001$ \\
\hline Normal & $156(68.42)$ & $87(32.10)$ & \\
\hline Increased & $72(31.58)$ & $184(67.90)$ & \\
\hline
\end{tabular}

$B M I$ body mass index, $W B C$ white blood cell, $C R P$ C-reactive protein, $O R$ odds ratio, $C I$ confidence interval, $C O P D$ chronic obstructive pulmonary disease

and found that the PII value was significantly correlated with the lymphocyte count, monocyte count, C-reactive protein level, procalcitonin level, days from illness onset, and body temperature. Li et al. [19] found that the severe/critical patients were older and showed higher incidence of comorbidities, cough, expectoration, chest pain, and dyspnea, and the incidences of consolidation, linear opacities, crazy-paving pattern, and bronchial wall thickening were higher in severe/ critical patients, while the incidences of lymph node enlargement, pericardial effusion, and pleural effusion were significantly higher than those in ordinary patients. Xiong et al. [20] found the CRP level, erythrocyte sedimentation rate, and lactate dehydrogenase level showed a significantly positive correlation with the severity of pneumonia assessed on initial CT, and the highest temperature and the severity of opacifications assessed on initial CT were significantly related to the progression of opacifications on follow-up CT. Although these studies explored some CT and clinical characteristics related to disease severity, they could not accurately and easily classify the CT findings.

LCA is a statistical method that takes advantage of unobserved, or latent, classes in the data that can be used to determine diagnostic performance characteristics [21].
LCA has recently been tested and found to be useful for identifying latent classes (subgroups) of radiological findings within multivariable datasets [22-24]. In this study, two radiological subgroups were identified based on the LCA of CT findings of patients with COVID-19 pneumonia. We found that peripheral distribution, GGO, maximum lesion range $\leq 5 \mathrm{~cm}$, involvement of 1-4 lobes, no air bronchogram, and no dilatation of vessels were more commonly in class 1 corresponding to the clinically moderate type. In contrast, diffuse distribution, mixture with GGO, crazy-paving pattern, consolidation, maximum lesion range $>10 \mathrm{~cm}$, involvement of 5 lobes, air bronchogram, and dilatation of vessels were more common in the class 2 corresponding to the clinically severe type. These CT characteristics are consistent with the findings of previous studies [25-31].

In the current study, noteworthy differences in clinical characteristics were observed between the LCA-derived subgroups. Older age, death, fever, hypertension, decreased lymphocyte count, and increased CRP levels were more common in class 2 . In the univariate analysis, we found that the older age, therapy of outcomes, presence of fever, presence of hypertension, decreased lymphocyte count, and increased CRP 
Table 4 The result of univariate analysis

\begin{tabular}{|c|c|c|c|}
\hline Variables & Statistics & OR $(95 \%$ CI $)$ & $p$ value \\
\hline \multicolumn{4}{|l|}{ Sex, $n(\%)$} \\
\hline Male & $258(51.70)$ & 1.0 (reference) & \\
\hline Female & $241(48.30)$ & $0.88(0.62,1.25)$ & 0.485 \\
\hline Age, mean (SD), years & $57.82 \pm 14.19$ & $1.04(1.02,1.05)$ & $<0.0001$ \\
\hline \multicolumn{4}{|l|}{ Age, $n(\%)$} \\
\hline$<60$ year & $248(49.70)$ & 1.0 (reference) & \\
\hline$\geq 60$ year & $251(50.30)$ & $2.17(1.52,3.11)$ & $<0.0001$ \\
\hline BMI, mean (SD), kg/m² & $22.68 \pm 3.16$ & $0.98(0.93,1.04)$ & 0.594 \\
\hline \multicolumn{4}{|l|}{ BMI, $n(\%)$} \\
\hline$<23 \mathrm{~kg} / \mathrm{m}^{2}$ & $248(49.70)$ & 1.0 (reference) & \\
\hline$\geq 23 \mathrm{~kg} / \mathrm{m}^{2}$ & $251(50.30)$ & $0.96(0.67,1.36)$ & 0.813 \\
\hline \multicolumn{4}{|l|}{ Clinical type, $n(\%)$} \\
\hline Moderate & $359(71.94)$ & 1.0 (reference) & \\
\hline Severe & $140(28.06)$ & $3.49(2.26,5.39)$ & $<0.0001$ \\
\hline \multicolumn{4}{|c|}{ Initial nucleic acid test, $n(\%)$} \\
\hline Negative & $30(6.01)$ & 1.0 (reference) & \\
\hline Positive & $469(93.99)$ & $0.90(0.43,1.90)$ & 0.789 \\
\hline \multicolumn{4}{|l|}{ Outcomes, $n(\%)$} \\
\hline Recovery & $422(84.57)$ & 1.0 (reference) & \\
\hline Therapy & $69(13.83)$ & $3.34(1.85,6.04)$ & $<0.0001$ \\
\hline Death & $8(1.60)$ & - & - \\
\hline \multicolumn{4}{|l|}{ Fever, $n(\%)$} \\
\hline No & $72(14.43)$ & 1.0 (reference) & \\
\hline Yes & $427(85.57)$ & $2.38(1.42,4.00)$ & 0.001 \\
\hline \multicolumn{4}{|l|}{ Cough, $n(\%)$} \\
\hline No & $113(22.65)$ & 1.0 (reference) & \\
\hline Yes & $386(77.35)$ & $1.28(0.84,1.95)$ & 0.250 \\
\hline \multicolumn{4}{|c|}{ Myalgia or arthralgia, $n(\%)$} \\
\hline No & $290(58.12)$ & 1.0 (reference) & \\
\hline Yes & $209(41.88)$ & $1.28(0.90,1.84)$ & 0.173 \\
\hline \multicolumn{4}{|l|}{ Headache, $n(\%)$} \\
\hline No & $486(97.39)$ & 1.0 (reference) & \\
\hline Yes & $13(2.61)$ & $0.98(0.32,2.96)$ & 0.973 \\
\hline \multicolumn{4}{|l|}{ Nausea or vomiting, $n(\%)$} \\
\hline No & $493(98.80)$ & 1.0 (reference) & \\
\hline Yes & $6(1.20)$ & $0.84(0.17,4.20)$ & 0.831 \\
\hline \multicolumn{4}{|l|}{ Diarrhea, $n(\%)$} \\
\hline No & $465(93.19)$ & 1.0 (reference) & \\
\hline Yes & $34(6.81)$ & $1.07(0.53,2.16)$ & 0.849 \\
\hline \multicolumn{4}{|l|}{ Abdominal pain, $n(\%)$} \\
\hline No & $494(99.00)$ & 1.0 (reference) & \\
\hline Yes & $5(1.00)$ & - & 0.981 \\
\hline \multicolumn{4}{|l|}{ Smoking history, $n(\%)$} \\
\hline No & $466(93.39)$ & 1.0 (reference) & \\
\hline Yes & $33(6.61)$ & $1.74(0.83,3.68)$ & 0.145 \\
\hline Cardiovascular disease, $n$ & & & \\
\hline No & $452(90.58)$ & 1.0 (reference) & \\
\hline Yes & $47(9.42)$ & $1.05(0.57,1.91)$ & 0.884 \\
\hline Diabetes, $n(\%)$ & & & \\
\hline No & $444(88.98)$ & 1.0 (reference) & \\
\hline Yes & $55(11.02)$ & $1.69(0.94,3.03)$ & 0.081 \\
\hline Hypertension, $n(\%)$ & & & \\
\hline No & $348(69.74)$ & 1.0 (reference) & \\
\hline Yes & $151(30.26)$ & $1.73(1.17,2.55)$ & 0.006 \\
\hline COPD, $n(\%)$ & & & \\
\hline No & $497(99.60)$ & 1.0 (reference) & \\
\hline Yes & $2(0.40)$ & $0.84(0.05,13.52)$ & 0.903 \\
\hline Chronic liver disease, $n(c$ & & & \\
\hline No & $488(97.80)$ & 1.0 (reference) & \\
\hline Yes & $11(2.20)$ & $1.01(0.30,3.35)$ & 0.987 \\
\hline Chronic renal disease, $n$ & & & \\
\hline No & $492(98.60)$ & 1.0 (reference) & \\
\hline Yes & $7(1.40)$ & $0.63(0.14,2.83)$ & 0.544 \\
\hline Malignant tumor, $n(\%)$ & & & \\
\hline No & $486(97.39)$ & 1.0 (reference) & \\
\hline
\end{tabular}


Table 4 (continued)

\begin{tabular}{lcll}
\hline Variables & Statistics & OR (95\% CI) & $p$ value \\
\hline Yes & $13(2.61)$ & $1.36(0.44,4.21)$ & 0.597 \\
WBC count, $n(\%)$ & & & \\
$\quad$ Decreased & $46(9.22)$ & 1.0 (reference) & $1.72(0.93,3.19)$ \\
Normal & $422(84.57)$ & $2.98(1.15,7.75)$ & 0.086 \\
Increased & $31(6.21)$ & 1.0 (reference) & 0.025 \\
Lymphocyte count, $n(\%)$ & & $2.24(0.44,11.27)$ & 0.329 \\
Increased & $8(1.60)$ & $7.68(1.51,39.19)$ & 0.014 \\
Normal & $288(57.72)$ & 1.0 (reference) & \\
Decreased & $203(40.68)$ & $4.58(3.14,6.69)$ & $<0.0001$ \\
CRP, $n(\%)$ & $243(48.70)$ & & \\
Normal & $256(51.30)$ & & \\
Increased & &
\end{tabular}

$B M I$ body mass index, $W B C$ white blood cell, $C R P$ C-reactive protein, $O R$ odds ratio, $C I$ confidence interval, $C O P D$ chronic obstructive pulmonary disease

levels were parameters significantly associated with an increased risk for class 2. To explore the relationship between the LCA-derived subgroups and clinically moderate and severe types, multivariable logistic models were used. In the crude model, minimally adjusted model, and fully adjusted model, significant associations were all found between the LCA-derived subgroups and clinical moderate and severe types. The patients with clinical severe type had a 1.97-fold higher risk of class 2 compared with the patients with clinical moderate type.

Our study had several limitations. First, it was a retrospective single-center study; second, interpretation of CT images was performed by consensus. Finally, we only included the clinical moderate and severe patients, which would lead to a potential inclusion bias.

\section{Conclusions}

Two radiological subgroups were identified based on the LCA of CT findings of the patients with COVID-19. The demographic and clinical differences between the identified subgroups were significantly different. The two radiological subgroups were significantly associated with clinical moderate and severe types.

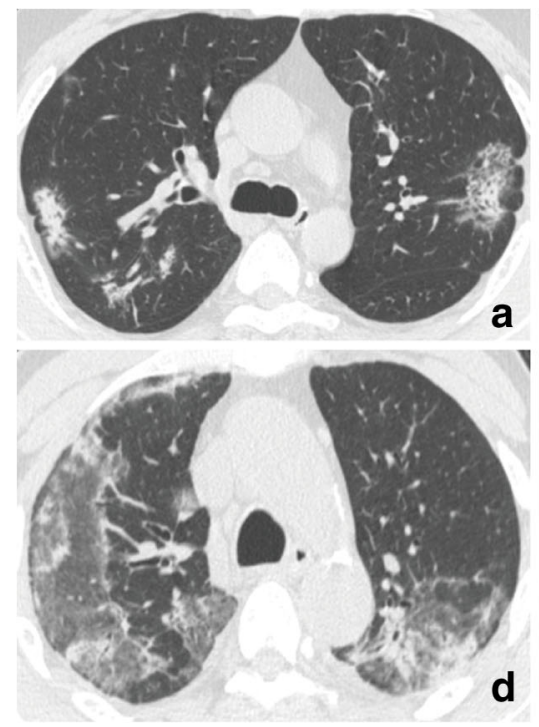

Fig. 4 a-c Chest CT findings of a 34-year-old man with moderate COVID-19 pneumonia a CT image of lung parenchyma showed multifocal crazy-paving pattern and consolidation peripherally distributed in the superior lobes of both lungs. b The lesions were automatically labeled by artificial intelligence software. $\mathbf{c}$ Three-dimensional volume-rendered reconstruction showed the extent of crazy-paving pattern and consolidation with scattered pattern. d-f Chest CT of a 68-year-old man with
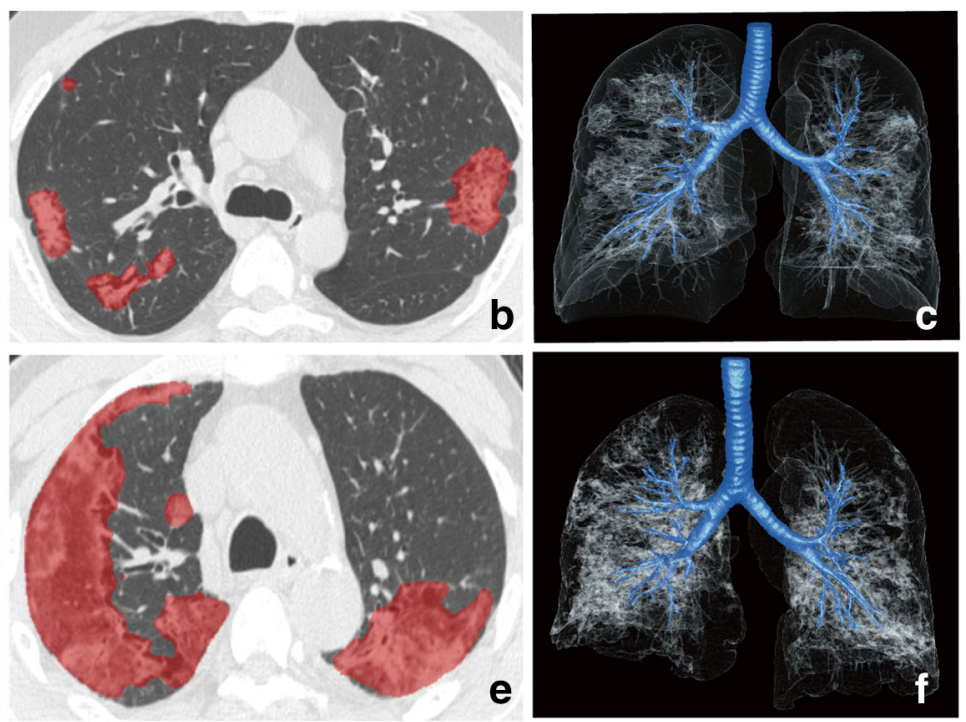

severe COVID-19 pneumonia. d CT image of lung parenchyma showed multi-focal GGO and consolidation diffusely distributed in the middle and inferior lobes of the right lung and of inferior lobe of the left lung. e The lesions were automatically labeled by artificial intelligence software. f Three-dimensional volume-rendered reconstruction showed the extent of the crazy-paving pattern and consolidation with a scattered pattern. GGO, ground glass opacity 
Table 5 Relationship between the clinical types and LCA-derived subgroups

\begin{tabular}{|c|c|c|c|c|c|c|}
\hline \multirow[t]{2}{*}{ Variable } & \multicolumn{2}{|l|}{ Model 1} & \multicolumn{2}{|l|}{ Model 2} & \multicolumn{2}{|l|}{ Model 3} \\
\hline & OR $(95 \% \mathrm{CI})$ & $p$ value & OR $(95 \% \mathrm{CI})$ & $p$ value & OR $(95 \% \mathrm{CI})$ & $p$ value \\
\hline \multicolumn{7}{|c|}{ Clinical types } \\
\hline Moderate & 1.0 (reference) & & 1.0 (reference) & & 1.0 (reference) & \\
\hline Sever & $3.49(2.26,5.39)$ & $<0.0001$ & $3.01(1.92,4.70)$ & $<0.0001$ & $1.97(1.09,3.54)$ & 0.025 \\
\hline
\end{tabular}

$O R$ odds ratio, $C I$ confidence, $L C A$ latent class analysis

Model 1: we did not adjust other co-variants

Model 2: we adjusted age, sex, and body mass index

Model 3: we further adjusted outcomes, fever, hypertension, lymphocyte count, C-reactive protein

Funding information This work was supported in part by National Science Foundation for Scientists of China (81871352) and National Science Foundation for Young Scientists of China (81701689).

\section{Compliance with ethical standards}

Guarantor The scientific guarantor of this publication is Yun Bian.

Conflict of interest The authors of this manuscript declare no relationships with any companies whose products or services may be related to the subject matter of the article.

Statistics and biometry No complex statistical methods were necessary for this paper.

Informed consent Written informed consent was waived by the Institutional Review Board.

Ethical approval Institutional Review Board approval was obtained by Huoshenshan hospital.

\section{Methodology}

- Retrospective

- Diagnostic or prognostic study

- Performed at one institution

\section{References}

1. Zhu N, Zhang D, Wang W et al (2020) A novel coronavirus from patients with pneumonia in China, 2019. N Engl J Med. https://doi. org/10.1056/NEJMoa2001017

2. Chen Y, Liu Q, Guo D (2020) Emerging coronaviruses: genome structure, replication, and pathogenesis. J Med Virol. https://doi. org/10.1002/jmv.25681

3. Chan JF, Yuan S, Kok KH et al (2020) A familial cluster of pneumonia associated with the 2019 novel coronavirus indicating person-to-person transmission: a study of a family cluster. Lancet. https://doi.org/10.1016/S0140-6736(20)30154-9

4. Phan LT, Nguyen TV, Luong QC et al (2020) Importation and human-to-human transmission of a novel coronavirus in Vietnam. N Engl J Med 382:872-874

5. Holshue ML, DeBolt C, Lindquist S et al (2020) First case of 2019 novel coronavirus in the United States. N Engl J Med. https://doi. org/10.1056/NEJMoa2001191
6. Giovanetti M, Benvenuto D, Angeletti S, Ciccozzi M (2020) The first two cases of 2019-nCoV in Italy: where they come from? J Med Virol. https://doi.org/10.1002/jmv.25699

7. Organization WH Clinical management of severe acute respiratory infection when novel coronavirus (2019-nCoV) infection is suspected. 2019. At https://www.who.int/csr/disease/coronavirus infections/InterimGuidance_ClinicalManagement NovelCoronavirus_11Feb13u.pd f ?ua $=1 \& u a=1$. Accesse $\bar{d}$ February 11, 2020

8. General Office of National Health Committee. Office of State Administration of Traditional Chinese Medicine. Notice on the issuance of a program for the diagnosis and treatment of novel coronavirus (2019-nCoV) infected pneumonia (trial seventh edition) (2020-03-4) http://bgs.satcm.gov.cn/zhengcewenjian/2020-03-04/ 13594.html

9. Organization WH WHO guidelines for the global surveillance of severe acute respiratory syndrome (SARS). 2004. At https://www. who.int/csr/resources/publications/WHO_CDS_CSR_ARO_ 2004 1.pdf?ua $=1$. Published January, 2004. Updated October, 2004

10. Organization WH Middle East respiratory syndrome case definition for reporting to WHO. 2017. https://www.who.int/csr/disease/ coronavirus_infections/mers-interim-case definition.pdf?ua $=1$

11. Azhar EI, El-Kafrawy SA, Farraj SA et al (2014) Evidence for camel-to-human transmission of MERS coronavirus. N Engl J Med 370:2499-2505

12. Franquet $\mathrm{T}$ (2011) Imaging of pulmonary viral pneumonia. Radiology 260:18-39

13. Koo HJ, Lim S, Choe J, Choi SH, Sung H, Do KH (2018) Radiographic and CT features of viral pneumonia. Radiographics 38:719-739

14. Harisinghani MG (2013) Atlas of lymph node anatomy. Springer, New York

15. H A (1974) A new look at the statistical model identification. IEEE Trans Autom Control 19:716-723

16. Iwasawa T, Sato M, Yamaya T et al (2020) Ultra-high-resolution computed tomography can demonstrate alveolar collapse in novel coronavirus (COVID-19) pneumonia. Jpn J Radiol 38:394-398

17. Wu J, Wu X, Zeng W et al (2020) Chest CT findings in patients with coronavirus disease 2019 and its relationship with clinical features. Invest Radiol. https://doi.org/10.1097/RLI. 0000000000000670

18. Albarello F, Pianura E, Di Stefano F et al (2020) 2019-novel coronavirus severe adult respiratory distress syndrome in two cases in Italy: an uncommon radiological presentation. Int J Infect Dis 93: 192-197

19. Li K, Wu J, Wu F et al (2020) The clinical and chest CT features associated with severe and critical COVID-19 pneumonia. Invest Radiol. https://doi.org/10.1097/RLI.0000000000000672 
20. Xiong Y, Sun D, Liu Y et al (2020) Clinical and high-resolution CT features of the COVID-19 infection: comparison of the initial and follow-up changes. Invest Radiol. https://doi.org/10.1097/RLI. 0000000000000674

21. Buijze GA, Mallee WH, Beeres FJ, Hanson TE, Johnson WO, Ring D (2011) Diagnostic performance tests for suspected scaphoid fractures differ with conventional and latent class analysis. Clin Orthop Relat Res 469:3400-3407

22. Scheltens NM, Galindo-Garre F, Pijnenburg YA et al (2016) The identification of cognitive subtypes in Alzheimer's disease dementia using latent class analysis. J Neurol Neurosurg Psychiatry 87: 235-243

23. Zwemmer JN, Berkhof J, Castelijns JA, Barkhof F, Polman CH, Uitdehaag BM (2006) Classification of multiple sclerosis patients by latent class analysis of magnetic resonance imaging characteristics. Mult Scler 12:565-572

24. Cowman SA, Jacob J, Obaidee $\mathrm{S}$ et al (2018) Latent class analysis to define radiological subgroups in pulmonary nontuberculous mycobacterial disease. BMC Pulm Med 18:145

25. Pan Y, Guan H, Zhou S et al (2020) Initial CT findings and temporal changes in patients with the novel coronavirus pneumonia (2019-nCoV): a study of 63 patients in Wuhan, China. Eur Radiol. https://doi.org/10.1007/s00330-020-06731-x

26. Pan F, Ye T, Sun P et al (2020) Time course of lung changes on chest CT during recovery from 2019 novel coronavirus (COVID-
19) pneumonia. Radiology. https://doi.org/10.1148/radiol. 2020200370:200370

27. Xie X, Zhong Z, Zhao W, Zheng C, Wang F, Liu J (2020) Chest CT for typical 2019-nCoV pneumonia: relationship to negative RTPCR testing. Radiology. https://doi.org/10.1148/radiol. 2020200343

28. Shi H, Han X, Zheng C (2020) Evolution of CT manifestations in a patient recovered from 2019 novel coronavirus (2019-nCoV) pneumonia in Wuhan, China. Radiology. https://doi.org/10.1148/radiol. 2020200269:200269

29. Lei J, Li J, Li X, Qi X (2020) CT imaging of the 2019 novel coronavirus (2019-nCoV) pneumonia. Radiology. https://doi.org/ 10.1148/radiol.2020200236:200236

30. Fang Y, Zhang H, Xu Y, Xie J, Pang P, Ji W (2020) CT manifestations of two cases of 2019 novel coronavirus (2019-nCoV) pneumonia. Radiology. https://doi.org/10.1148/radiol.2020200280: 200280

31. Duan YN, Qin J (2020) Pre- and posttreatment chest CT findings: 2019 novel coronavirus (2019-nCoV) pneumonia. Radiology. https://doi.org/10.1148/radiol.2020200323:200323

Publisher's note Springer Nature remains neutral with regard to jurisdictional claims in published maps and institutional affiliations. 